\title{
A Review on Wireless Data Center Management
}

\author{
Neeraj Kumari \\ M.Tech. Research Scholar \\ Department of Computer Science \& Engineering \\ Krishna Engineering College, Ghaziabad, INDIA
}

\author{
Arun Agarwal \\ Assistant Professor \\ Department of Computer Science \& Engineering \\ Krishna Engineering College, Ghaziabad, INDIA
}

\begin{abstract}
Data Center Management is an important challenge in the fast era to fulfill ever-evolving computational demand around cloud computing, big data and infrastructure. DC (Data Center) are used to resolve the issues related to overutilization of resource, application failures, data security, power usage effectiveness and infrastructure costs which requires proactive solutions that are business intelligent and built over a network of sense-points with the help of sensor networks that are suitable to deliver reliable trends. Sensor networks with telemetry and control functions is used for collection \& delivering of the data by improved server rack utilization, improved DC cooling and load balancing with dynamic capping is used. SCADA system is used with sense-point data to process the information wireless sensor networks in DCs comprises sensor nodes, gateways, routers, server platforms and software application for the overall management of various application.
\end{abstract}

\section{Keywords}

Wireless Sensor Networks (WSN), Supervisory Control and Data Acquisition (SCADA), Gateway, Genetic Algorithm (GA), Sub Networks, Data Center (DC), Power Usage Effectiveness (PUE).

\section{INTRODUCTION}

Recent advances data centers (DCs) are important challenge to fulfilling ever-evolving computational demands around cloud computing, big data, and this infrastructure. To operate a DC, power supplies, network connections, environmental controls (e.g., air conditioning, humidity) and security infrastructure are needed. Technology and business challenges such as virtualization, load consolidation, real-time troubleshooting, and service-level guarantees require a robust and adaptive server management plan for enterprise. Data centers have emerged to be an increasingly important computing infrastructure. The wireless sensor networks can provide fine-grained measurements in data centers, and achieve better control of the data center platform for energy efficiency. The DC issues are

related to overutilization of resources, application failures, data security, power usage effectiveness (PUE), and infrastructure costs. It requires proactive solutions that are business intelligent and built over a network of sense points that are guaranteed to deliver reliable trends and measurements in a reliable and timely fashion. Since it is expensive to build new DCs, the best option is to improve usage of an existing facility through lower infrastructure overhead to deliver better resource management. Sensor networks with telemetry and control functions plays an important role for the management (collection \& delivering) of the data by improved server rack utilization, improved load balancing through dynamic capping of thermally constrained systems rather than to establish a new wireless Data Center on the infrastructure front, DCs face considerable challenges in seamless integration of telemetry and control functions. These functions are essential in management tasks related to power capping, cooling, reliability, predictability, survivability, and adaptability control. In this review paper one wireless Data Center Management (collecting and transmitting) sensor information access WSN Learning methods such as a genetic algorithms, a Machine Learning approach is used for synthesis of data Gateway approach is used to monitor and to analyze data collection from individual nodes to establish various routes for better performance Khanna et al. ${ }^{[1]}$ The sense-point data and environmental data is delivered to the supervisory control and data acquisition (SCADA) system in a guaranteed duration to monitor, consolidate, and analyze real-time process control data. The SCADA system uses the sense-point data to produce models and tools for facility management and performance optimization (Fig1). In this figure it is divided into three parts

1. Monitor (monitoring parameters are cooling, power budgeting, thermal variance, load balancing, server performance).

2. Analyze and Present (data collection, classification, archiving, presentation, and analytics).

3. Control (monitoring, workload, scheduling, airflow/cooling, control, failure, prediction, dynamic server, provisioning).

Wireless system also provides flexibility to grow and adapt as a DC evolves over time. 


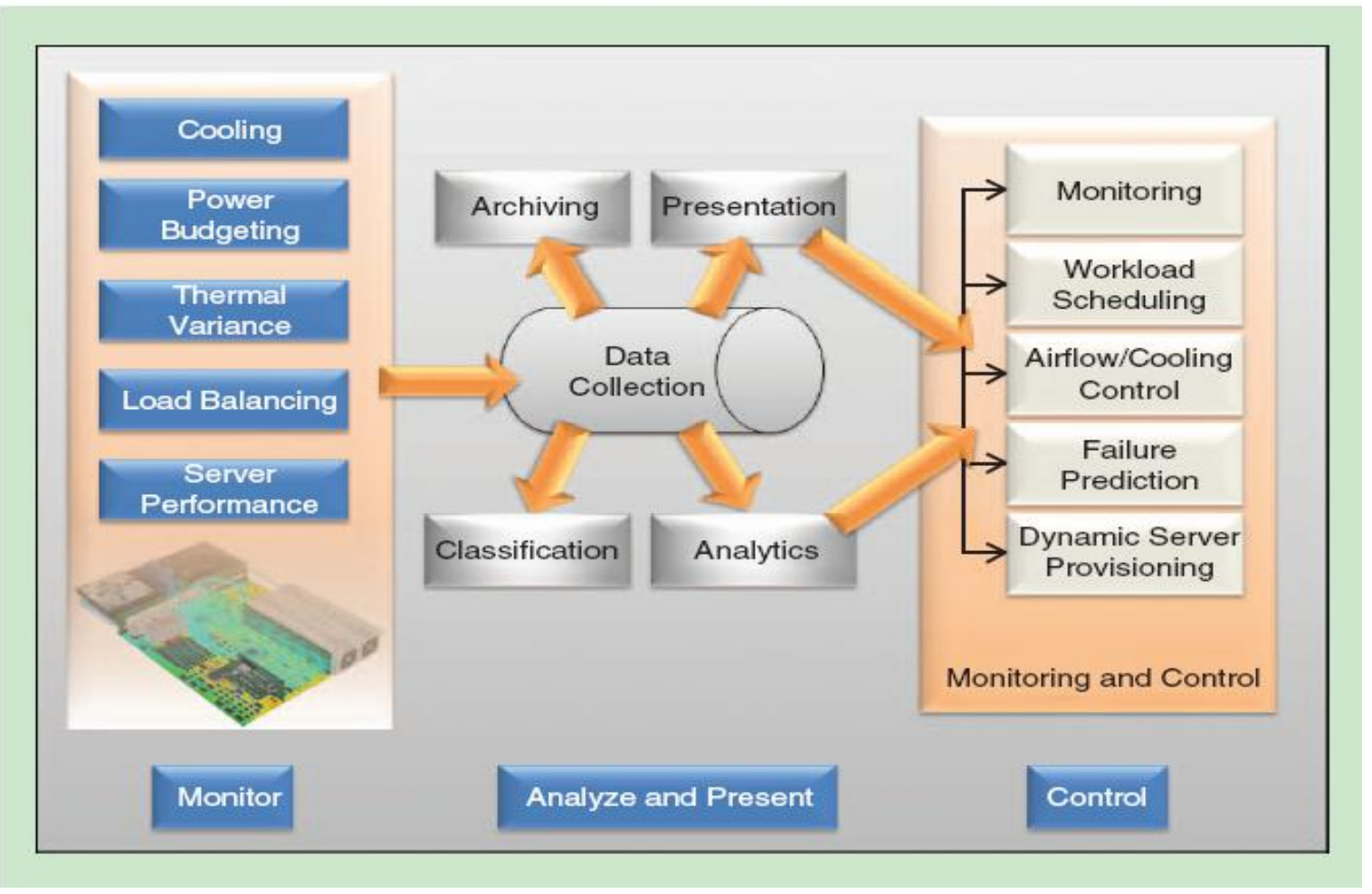

Fig1. Block Diagram of Data Management Techniques in WSN

Wireless Sensor Networks (WSNs) are usually selforganizedDifferent types of pros and cons of the technologies used andwireless ad hoc networks comprising of a large number ofcomponents are shown in table1.resources constrained sensor nodes.

Feng Wang et al. ${ }^{[13]}$ one of the most important tasks of these sensor nodes are systematic collection of data and transmits gathered data to a distant base station (BS). It minimizes the energy consumption involved by communication in wireless sensor networks.

Khanna, R. ${ }^{[15]}$ Genetic Algorithm reduced complexity for optimization of multi-hop sensor networks. Yogeswari et al. [16] DCs has most important applications in sensor data collection as it collects the environment data from all sensor nodes periodically.

Wireless Data Center Management provides facilities to determine current energy cost, energy savings potential, Ease of installation, maintenance of technology, cost-effectiveness, customer satisfaction, energy issues associated with the data center and potential solutions, improvement of airflow by reducing supply and return air mixing, improved cooling efficiency, continuous optimization of energy efficiency measures, balance data center IT (Information Technology) equipment load to the data center cooling capacity to increase space utilization and capacity.

\section{WSN INFRASTRUCTURE}

Data centers have become increasingly important in today's computing technology, and a number of sensor networks have been developed for this critical infrastructure. In today's era DC (data center), wireless sensor network can act as low-cost candidate (ten times) for monitoring tasks as it is nonintrusive, can provide wide coverage, and can be easily re-purposed. Within a DC, a WSN system clusters a network of sensor devices that enables real-time monitoring to observe and manage energy, thermal, and performance constraints. Feng et at ${ }^{[5][13]}$ The WSN is useful in debugging tool to monitor hot spots, benchmarking, and system forensics, including alerts and alarms. ZigBee et al ${ }^{[16][17]}$ Wireless Sensor Networks are infrastructure contains sensing, computing and communication elements that aim to give its controllers the ability to measure, collect and react to occurrences in the monitored environment. Above idea is to develop a wireless monitoring infrastructure that can fulfill the following characteristics:

- Ability to reduce interference and noise while operating in a dense wireless network

- Ability to optimize sensor data flow for efficient battery utilization using network load balancing and route-delay optimization.

Table 1. Different pros and cons of the Technology and Components

\begin{tabular}{|l|l|l|}
\hline $\begin{array}{l}\text { Technology } \\
\text { /Components }\end{array}$ & PROS & CONS \\
\hline 1.Thermal Monitoring & $\begin{array}{l}\text { Minimize hot spots or } \\
\text { optimize cooling }\end{array}$ & Expensive \\
\hline $\begin{array}{l}\text { 2.Wireless Sensor } \\
\text { Network }\end{array}$ & $\begin{array}{l}\text { Stores limited source } \\
\text { of energy, and no } \\
\text { constraint of cables } \\
\text { and has mobility }\end{array}$ & $\begin{array}{l}\text { It is to } \\
\text { disturb the } \\
\text { propagation } \\
\text { of waves } \\
\text { and hack } \\
\text { your } \\
\text { networking } \\
\text { and it is too } \\
\text { costly to use }\end{array}$ \\
\hline $\begin{array}{l}\text { 3.Sensor Network } \\
\text { Synthesis }\end{array}$ & $\begin{array}{l}\text { Result would be error } \\
\text { free data by GA. }\end{array}$ & $\begin{array}{l}\text { Delay } \\
\text { occurs. }\end{array}$ \\
\hline 4.Sub-network & Better performance of & Complex \\
\hline
\end{tabular}




\begin{tabular}{|c|c|c|}
\hline Synthesis & $\begin{array}{l}\text { networks for the } \\
\text { transmission }\end{array}$ & $\begin{array}{l}\text { Structure } \\
\text { and } \\
\text { expensive in } \\
\text { use. }\end{array}$ \\
\hline $\begin{array}{l}\text { 5.Sensor-Data } \\
\text { Collection: Protocol }\end{array}$ & $\begin{array}{l}\text { Transmission rate } \\
\text { increases, } \\
\text { unidentified cannot } \\
\text { interfere the original } \\
\text { signals. }\end{array}$ & Costly \\
\hline 6. Route Fitness (FR) & $\begin{array}{l}\text { Data transmission } \\
\text { time becomes less }\end{array}$ & - \\
\hline 7. Gateways & $\begin{array}{lr}\text { Improve the data } \\
\text { throughput, eliminate } \\
\text { information } \\
\text { redundancy, } \\
\begin{array}{lr}\text { exploit and } \\
\text { information } \\
\text { compression }\end{array} \\
\end{array}$ & - \\
\hline 8. Genetic Algorithm & $\begin{array}{l}\text { Minimize the energy } \\
\text { consumption involved } \\
\text { by communications, } \\
\text { error free data, } \\
\text { Over-all system } \\
\text { performance } \\
\text { improves. }\end{array}$ & - \\
\hline
\end{tabular}

\section{RELATED WORK AND TECHNOLOGY \\ 3.1 Data Center: Thermal Monitoring in Dynamic Environment}

Data Centers have become a critical computing infrastructure in the era of cloud computing and DC works in a highly dynamic environment. In this environment, hot spots can be created as a result of temporal events [(e.g., increased workload on a set of servers) or spatial events (inefficiency of the computer room air conditioner (CRAC) units in delivering requisite cooling to a particular region in the DC].

The primary source of heat generation is chip or the system-onchip (SOC) in the heart of the platform is Microprocessor. On die sensors measures heat production in the chip and platform cooling devices such as fans which calibrate air flow accordingly to provide cooling.

Khanna et al. ${ }^{[1]}$ for example, an optimum decision are one that produces successive thermal snapshots with progressive diminishment of hot spots. Most prediction algorithms are based on periodic sampling of the states of several entities at once, the

DC's sensor network, sensors within servers, the DC's cooling infrastructure, cooling devices (e.g., fans) within servers, and finally, the set of workload running on the servers. The prediction logic is hence a discrete-time system, and it predicts the temperature rise in the $i^{\text {th }}$ platform at a given sampling instant $T$ as in equation (1) shown below:

$\dot{\varnothing}(\mathrm{T})=\sum_{\mathrm{t}=\mathrm{T}-\mathrm{d}}^{\mathrm{T}} \mu \mathrm{t} P(\mathrm{t})+\sum_{\mathrm{t}=\mathrm{T}-\mathrm{d}}^{\mathrm{T}} \omega \mathrm{t} \mathrm{T}(\mathrm{t})-\sum_{\mathrm{t}-\mathrm{T}-\mathrm{d}}^{\mathrm{T}} \lambda \mathrm{t} \mathrm{C}(\mathrm{t})$.

The first term on the right pertains to the accumulated power consumed by the running workload $(P)$, which causes a rise in the junction temperature of the component, and is eventually dissipated as heat. The second term pertains to the effects of the local environment $(T)$ in which the server is running, e.g. the effect of a hot spot caused by other racks or other equipment in the vicinity of the server or the redundant cooling delivered by an over-calibrated CRAC unit. The third term refers to the cooling performed purely by the server's cooling system $(C)$, e.g. convective cooling or liquid cooling. The constants $\mu \mathrm{t}, \omega \mathrm{t}$ and $\lambda t$ must be evaluated at each sampling period and adjusted to reflect the latest thermal snapshot of the system. e.g. direction of flow of heat from hot spots is in Fig 2.

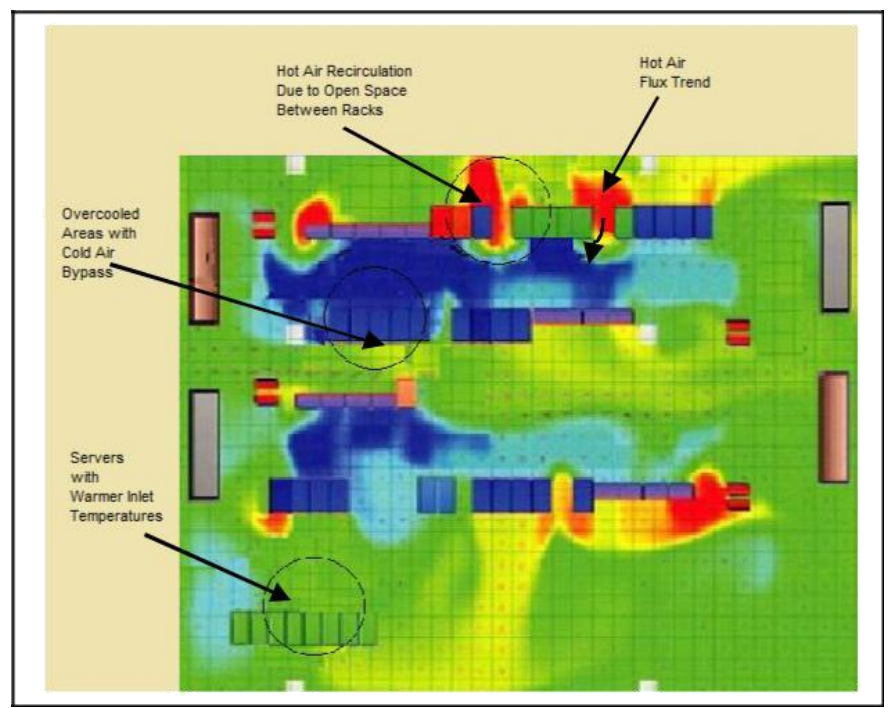

Fig 2. A thermal snapshot of the DC. Efficient monitoring and sense data yield is essential for capturing the accurate heat map.

\subsection{Data Center: Wireless Sensor Network Usage}

Wireless Sensor Networks (WSN) consists of several sensor enable nodes which are distributed in an environment and use batteries as energy resource and the idea of sensor networks based on collaborative effort of a large number of nodes. Khanna et al. ${ }^{[1]}$, ZigBeeet al. ${ }^{[19][20]}$ The noninvasive wireless sensors can measure and synthesize historical trends for air temperature, humidity air particle count, current, power, node utilization, workload performance, service-level agreements (SLAs), and other sense data. Different types of attributes of the WSN are related to auto-discovery, addressability, event signaling, uniqueness, abstraction model, and grouping.

The wireless sensor technology in a DC comprises sensor nodes, gateways, routers, server platforms, and software applications (similar to DC Manager) and establishes baseline energy consumption and identifies improvement opportunities by efficient provisioning and loading of server resources. Model relationship between server performance characteristics, energy consumption, and environmental parameters (temperature, humidity, subfloor pressure, etc.) and using monitoring infrastructure, develop automation strategy that performs adaptive workload provisioning (loading, off-loading, migrating, consolidating, etc.), air-flow control, and air-conditioning control. It uses monitoring infrastructure, develop automation strategy that performs adaptive workload provisioning (loading, offloading, migrating, consolidating, etc.), air-flow control, and air-conditioning control. 
Gutierrez et al. ${ }^{[18]}$ Time, frequency, and physical diversity are incorporated to assure reliability, scalability, power source flexibility, and ease of use. The sensor nodes are ultra-low power wireless transceivers that transfer data to and from integrated sensors or controllers. Fig 3 shows Reliability Wireless Sensor Networks.

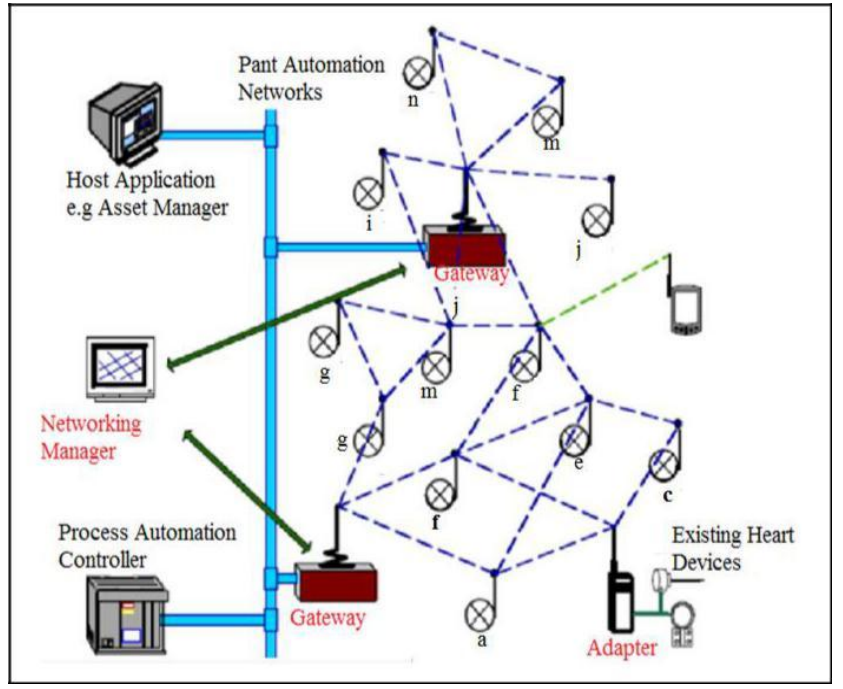

Fig 3. Reliability in Wireless Sensor Network

The ability of a sensor node to maintain the sensor network functionalities without any interruption caused due to sensor node failures can be considered as a measure of its reliability.

There are many reasons where sensor nodes fail. For example they may fail due to the lack of energy, due to physical damage, inactivity, a communication problem and even environmental interference.

These transceivers play a coordinated optimization role with neighboring nodes to eliminate operational interference. In this case the data latency is minimized.

\subsubsection{Sensors and Gateways}

Data Center WSN is a hierarchy of sensors, gateways, data sink, and data analyzers. Walia et al. ${ }^{[9]}$ Sensor devices support wireless communication, computation, and sensing. Sensors are edge devices that collect the data related to thermal power, performance, locality, and airflow information and transmit that intermediary between a sensor/router and a data sink. Gateways are improving the data throughput, eliminate information redundancy, and exploit locality information for information compression.

\subsubsection{Handling Sensor Data: Building Collection Trees}

Collection trees forms the basic building blocks of the sensor networks and the related applications. But collection trees working through traditional network protocols suffer from low delivery ratio. Couto et al. ${ }^{[2]}$ proposed an expected transmission count measure to find high-throughput paths on multi-hop wireless networks, which minimizes the expected number of packet transmissions required to successfully deliver a packet to its ultimate destination. Burri et al. ${ }^{[3]}$ propose the protocol that coordinates media access control (MAC)-layer, topology control, and routing to construct energy efficient communication
Subsystem. Madden et al. ${ }^{[4]}$ proposed protocol that enables simple, declarative queries for efficient distribution and execution in low-power WSNs. Koala ${ }^{[5]}$ proposed low duty cycles architecture that exploits the sensor-node idle periods to allow longer sleep times and proactively wakes them up upon bulk data download.

Ganesan et al. ${ }^{[6]}$ proposed a joint optimization scheme for sensor placement and transmission structure for data gathering. Sensor nodes are placed in a field such that they aid in minimizing communication energy while re-constructing sensed data at a sink within specified distortion bounds. Jie et al. ${ }^{[7]}$ describe RACNet innovative reliable data collection protocol (rDCP) data collection protocol for high throughput and high reliability data collection using similar concepts of channel diversity and bidirectional collection trees. Lui et al $\left.{ }^{[12}\right] \mathrm{A}$ general scheme that uses a machine learning approach for channel allocation using fitness function that incorporates attributes related to uniformity in allocations, number of hops, route balancing, router density, congestion aware reallocation, data patterns, proximity patterns, and sampling uniformity. A Machine Learning approach can facilitate sensor network provisioning and re-organization to reduce single-hop sensor node density through synthesizing interference free subnetworks for real-time data collection with latency constraints. This will require an approach to building optimal number of sub-networks for sensor data collection, and data-collection protocol for an individual sub-tree using time-slot allocation.

\subsection{Data Center Sensor Network Synthesis}

A genetic algorithm (GA) facilitates allocation of each wireless sensor node to one of the $N$ sub-network containers by minimizing the overall interference between neighboring nodes, while improving the packet delivery. Furthermore, parallel subnetworks would be synthesized which operate independently without any interference from other trees. It allows thinning of the dense sensor network and reduction of the average number of nodes that are within one-hop communication range. Goldberg et al. ${ }^{[8]} \mathrm{A}$ GA is one such stochastic search technique that resembles the natural evolution. This approach evolves optimal number of sub-networks through multichannel node allocation in a manner that minimizes the interference while meeting the data latency guarantees, thereby improving the data collection efficiency.

\subsection{Sub-Network Synthesis: A Machine Learning Approach}

Sub Network Synthesis, Mekkaoui et. al. ${ }^{[14]}$ GA-based approach to configure the randomly deployed sensors in a DC into an optimal number of non-interfering independent sub-networks with optimal routes and sensor membership. In the "Operational

Fitness: Re-configuration of Sub-networks" section, each subnetwork parallelize the data collection from its member sensors and sends them to the target in a compressed manner via the most cost-effective route. "Measuring the Quality of Subnetwork," to calculate of overall fitness and goal related to: a) reduced channel interference, $b$ ) balanced routing, c) operational efficiencies, and d) data collection efficiency.

In the modern DCs, a WSN acts as low-cost candidate (ten times) for monitoring tasks as it is non-intrusive, which provide wide coverage, and can be easily re-purposed. Each network will be further divided into multiple clusters that are separated by non-interfering characteristics. 


\subsection{Sensor-Data Collection: Protocol}

DATA_SEND Protocol minimizes the amount of messages that need to traverse through the tree. Data collection protocol is initiated by the gateway by traversing the DATA_SEND message to all the nodes sequentially on the parallel tree (trees on separate channels). Data is collected in depth-first manner and cached into the parent before transmitting up-stream. Data is collected in depth-first manner and cached into the parent before transmitting up-stream. These measurements are delivered upstream to the gateway as training data for executing GA function Each WAIT_SLOT define a node can transmit a collection of all down-stream sensor node packets.

A parent node enumerates all the children nodes according to number of nodes downstream. Parent traverses through enumerated children to send WAIT_SLOT threshold and sequence number through DATA_SEND message. This threshold identifies the earliest time-slot the children nodes can communicate with the parent (with measurement data).

This technique of channel distribution is to be optimized for minimizing interference and not for load balancing.

\subsection{Route Fitness (FR): Synthesizing Balanced Routes}

The GA measures the sensor-data trip-delay performance that leads to channel re-allocation to construct balanced routes. Khanna et al. ${ }^{[1]}$ sensor-nodes generate several kilo-bits of burst data in one sampling period and the fitness measures to allow balance loading through the network to minimize datatransmission latencies. Liu et al ${ }^{[11][12]}$ First, each node builds profile structure that contains information regarding all other nodes. In this technique the channel distribution optimizes for minimizing interference and not for load balancing.

The GA measures the data transmission efficiency that leads to optimal trade-offs between data compression opportunities, channel interference and balanced routes.

Liu et al. ${ }^{[10]}$ a key observation here is that the availability of sensor data using WSN in a data center is a cost effective solution to achieve accurate decision making.

\subsubsection{Sensor Network Performance}

GA synthesizes a solution that assists in maximizing the average single-hop distance by optimally allocating non-adjacent channel to Sensor Nodes Gateway allocates timeslots for interference free data collection, for nodes that share same channel and are within one-hop distance. The Gateway monitor analyzes the data collection trends and patterns from each individual nodes as well as established routes.

\section{CONCLUSION AND FUTURE WORK}

Data Center Management within time limitness with integration of various telemetry control functions, real- time monitoring and control of DC resource needs high fidelity monitoring and environmental trends. Wireless Sensor Networks for DCs management presents cheaper and better networks by using network topology evolutionary algorithm. GA is an intelligent system applied on every node. For better performance proper channel allocation has been used. A Machine Learning approach acts as a feedback tool for the Dc management. In this Wireless Data Center Management there is lot of future scope as per requirement of today era in form of performance, speed and reliability of Data Center Management. In this paper a lot of future scope is there for the improvement performance of the system.

\section{REFERENCES}

[1] RahulKhanna,HuapingLiu,andThanunathanRangarajan,"wi reless data center management" inProc Supplement 15273342/14@2014 IEEE S45, November/December 2014.

[2] D. S. J. D. Couto, D. Aguayo, J. Bicket, and R. Morris, "A high throughput path metric for multi-hop wireless routing, " in Proc. 9th Annu. Int. Conf. Mobile computing networking, San Diego, CA, Sept. 2003.

[3] N. Burri, P. von Rickenbach, and R. Wattenhofer, "Dozer:

[4] Ultra-low power data gathering in sensor networks," in Proc. 6th Int. Conf. Information processing sensor networks, ACM, New York, 2007, pp. 450-459.

[5] S. Madden, M. J. Franklin, J. M. Hellerstein, and W. Hong,"TAG:A Tiny Aggregation service for ad-hoc sensor networks," in Proc. 5th symp. ACM SIGOPS Operating Systems Review, Dec. 2002, vol. 36, pp. 131-146.

[6] R.-E. Musaloiu, C.-J. M. Liang, and A. Terzis, "Koala: Ultra-low power data retrieval in wireless sensor networks," in Proc. Int. Conf. Information Processing Sensor Networks, St. Louis, MO, 2008, pp. 421-432.

[7] J. Liu, F. Zhao, J. O'Reilly, A. Souarez, M. Manos, C.-J. M. Liang, and A. Terzis. Project Genome: Wireless sensor network for data center cooling. [Online]. Available: http://msdn.microsoft.com/en-us/library/ dd393313.aspx.

[8] D. Ganesan, R. Cristescu, and B. Beferull-Lozano, "Powerefficient sensor placement and transmission structure for data gathering under distortion constraints," J. ACM Trans. Senor Netw., vol. 2, no. 2, pp. 155-181, May 2006.

[9] D. Goldberg, Genetic Algorithm in Search, Optimization and Machine Learning. Boston, MA: Addison-Wesley, 1989.

[10] Nisha devi Vishal walia, Dr.Rahul malhotra "wireless Sensor networks are used to route sensor measurements to gateways and data centers to enable efficient plant management." Issn 2348-5426 international journal of advances in science and technology (ijast) vol 2 issue 3(September 2014).

[11] R. Khanna, H. Liu, and H. H. Chen, "Dynamic optimization of secure mobile sensor networks: A genetic algorithm," inProc. IEEE Int. Conf. Communications, June 2007.

[12] R. Khanna, H. Liu, and H. H. Chen, "Self-organization of Sensor networks using genetic algorithms," in Proc. IEEE Int. Conf. Communications,Istanbul, Turkey, June 2006, vol. 8, pp. 3377-3382.

[13] R. Khanna, H. Liu, and H. H. Chen, "Dynamic optimization of secure mobile sensor networks: A genetic algorithm," inProc. IEEE Int. Conf. Communications, June 2007, pp. 3413-3418.

[14] Feng Wang "Networked Wireless Sensor Data . .Collection: Issues, Challenges, and Approaches"Feng Wang, Student Member, IEEE, and Jiangchuan Liu, Senior Member, IEEE".

[15] Mekkaoui Kheireddine, Rahmoun Abdellatif, Gianluigi Ferrari "Genetic Centralized Dynamic Clustering in Wireless Sensor Networks" I. J. Computer Network and Information Security, 2015, 8, 1-8 Published Online July 
2015 in MECS (http://www.mecs-press.org/) DOI: . 10.5815/ijcnis.2015.08.01.

[16] Khanna, R., Liu, H. and Chen, H-H. (2006) 'SelfOrganization of sensor networks using genetic algorithms',Int. J. Sensor Networks, Vol. 1, Nos. 3/4, pp.241-252.

[17] R. Yogeswari, V.Subathra "A SURVEY ON EFFICIENTDATA COLLECTION IN WIRELESS SENSOR. NETWORKS" International Journal of Innovative Research in Computer and Communication Engineering (An ISO 3297: 2007 Certified Organization) Vol. 1, Issue 9, November 2013.
[18] A True System-on-Chip Solution for 2.4-GHz IEEEand ZigBee Applications, IEEE Standard 802.15.4.

[19] J. A. Gutierrez, L. Winkel, E. H. Callaway, Jr., and R. L. Barrett, Jr.,Low-Rate Wireless Personal Area Networks: Enabling Wireless Sensors with IEEE 802.15. 4.Piscataway, NJ: IEEE Standards Assoc., 2011.

[20] ZigBee Alliance, “Zigbee specification," ZigBee Document053474r20, Revision 5, Sept. 12, 2012.

[21] ZigBee Alliance, "ZigBee RF4CE specification," ZigBeeDocument 094945r00ZB, Version 1.01, Jan. 2010. 